\title{
Research on the Weak Line Identification Method of Energy Consumption in 10kV Distribution Network
}

\author{
Wei Fu ${ }^{a}$, Haifeng Huang ${ }^{\mathrm{b}}$ and Lijun $\mathrm{Ma}^{\mathrm{c}}$ \\ State grid Hubei energy service Co., Ltd., Wuhan, 430077, China \\ a283727819@qq.com, b181259409@qq.com, ' $196888590 @ q q . c o m$
}

Keywords: Energy consumption, weak line identification, loss indexes, loss intervals.

\begin{abstract}
A weak line identification method of energy consumption, which is suitable to the $10 \mathrm{kV}$ distribution network, is proposed. First, clearly put forward six loss indexes of $10 \mathrm{kV}$ distribution network, so as to build a distribution network weak line index system. Second, on the basis of six indexes, divide distribution network energy consumptions into 4 loss intervals. Comparing measurement and calculation loss in actual running and loss intervals to estimate which interval the loss is in. This process is the initial judgment. Finally to make sure the weak line the root cause analysis in the interval is carried out, which realize the identification of weak line in distribution network. This method helps transforming the energy consumption value which is difficult to identified, into certain scope of the weak line identification of energy consumption, provides scientific guidance for the actual running.
\end{abstract}

\section{Introduction}

In China, government pays high attention to energy conservation transformation of distribution network. It is necessary to excavate the weak line of high energy consumption. So energy consumption statistics is the basis and key of the weak line excavation. The common energy consumption calculation methods used in $10 \mathrm{kV}$ distribution network have many drawbacks, such as calculation cycle is very long, calculation assumptions do not agree with the actual running state, etc. At the same time, there are many problems in $10 \mathrm{kV}$ distribution network that lack of measurement devices, low level of automation, lack of effective statistical measurements.

Theory calculation methods such as equivalent hours of maximum loss method, root-mean-square current method and energy losses calculation guide rule in power grid, roughly calculate the energy consumption in distribution network. These methods assume that electric loads are not changed over a period of time, which is obviously not match the situation of actual running. So the calculation results of these methods are not accurate enough to guide the loss reduction. Meter reading monitor statistic need high cost of investment, operation maintenance, and easily affected by many factors, such as the bad monitoring results, error artificial meter reading, and error meter ratio and communication failure. It is also cannot help calculating the energy consumption accurately. In conclusion, this paper will research a method that changes power loss calculation into weak line of energy consumption excavation. So as to effectively reflect the actual situation of energy consumption of distribution network, which is beneficial to guide the transforming work of energy conservation and loss reduction.

\section{Weak Line Identification Index System}

\subsection{Weak Line Identification Index}

There are six indexes set to be the weak line identification index of distribution network.1) Maximum power loss electric quantity and maximum line loss per unit; 2) Guideline loss electric quantity and guideline line loss per unit; 3) Minimum loss electric quantity and minimum line loss per unit; 4) Zero reactive loss electric quantity and zero reactive line loss per unit 5) Three-phase current harmonic total cumulative rate; 6) Un-balanced load cumulative rate. 
(1) Maximum power loss electric quantity

When power supply head end current is a determined value, there is a load distribution mode that measured and calculated line loss is the maximum value. Its corresponding loss of electricity is the maximum loss electric quantity.

(2) Guideline loss electric quantity

When power supply head end current is a determined value, there is a load distribution mode that determined by the guide rule in which every distribution transformers load coefficients are the same. Its corresponding loss of electricity is the guideline loss electric quantity.

(3) Minimum loss electric quantity

When power supply head end current is a determined value, there is a load distribution mode that measured and calculated line loss is the minimum value. Its corresponding loss of electricity is the minimum loss electric quantity.

(4) Zero reactive loss electric quantity

When power supply head end current is a determined value, assume that after the ideal of reactive power compensation, the head end current reactive component is zero. After the head end current subtract the reactive current, use the guideline [] in which every distribution transformers load coefficients are the same to determine the load distribution mode. Its corresponding loss of electricity is the zero reactive loss electric quantity.

[1]- [4] Each loss electric quantity divides the head end current can get each line loss per unit.

[5] Three-phase current harmonic total cumulative rate

The percentage of loss electric quantity produced by harmonic current and guideline loss electric quantity (fundamental harmonic) is three-phase current harmonic total cumulative rate.

[6] Un-balanced load cumulative rate

The percentage of additional loss electric quantity caused by three-phase unbalanced load and guideline loss electric quantity (balanced three-phrase load) is the un-balanced load cumulative rate.

\subsection{Maximum and Minimum Energy Consumption Intervals Calculation Method}

There is a power-flow distribution way to make the power loss to the maximum, and also a power-flow distribution way to make the power loss to the minimum in the distribution network. Use node resistance matrix to solve constrained optimization problems of load power losses in distribution network. By solving the maximum and minimum equivalent resistance curves, finally calculate the maximum and minimum energy consumption within a given period of time in $10 \mathrm{kV}$ distribution network.

1) Distribution network topology analysis

Electric transmission lines and transformers are the main power consumption components, other equipment, such as, capacitors, mutual inductors, and control and protect equipment, their power losses take a very small proportion. For this reason, distribution network topology is built by the transmission lines, transformers' resistances and the distribution network structure.

2) Distribution network equivalent resistance analysis

According to distribution network topology, assume that the head end is earth connected in distribution network, then conductance matrix $G$ can be made. Distribution network equivalent resistance matrix $\mathrm{R}$ is G's inverse matrix:

$$
R=G^{-1}=\left[\begin{array}{cc}
R_{n} & R_{n m} \\
R_{m n} & R_{m}
\end{array}\right]
$$

Where $\mathrm{n}$ is the number of load nodes in a distribution network, $\mathrm{m}$ is the number of contact nodes, load nodes numbers are prior to contact nodes numbers. $\mathrm{Rn}$ is a square matrix with $\mathrm{n}$ rows and $\mathrm{n}$ columns; diagonal elements are the self-resistance of load nodes, off-diagonal elements are the mutual resistances between load nodes.

3) Distribution network loss power analysis

Assume that the injection currents of each load nodes are $\left[I_{1}, I_{2}, \cdots \cdots, I_{n}\right]$.

Distribution network loss power $\mathrm{P}$ is: 


$$
P=\left[\begin{array}{l}
I_{1} \\
\vdots \\
I_{n} \\
0 \\
\vdots \\
0
\end{array}\right]^{T} \bullet\left[\begin{array}{cc}
R_{n} & R_{n m} \\
R_{m n} & R_{m}
\end{array}\right] \bullet\left[\begin{array}{l}
I_{1} \\
\vdots \\
I_{n} \\
0 \\
\vdots \\
0
\end{array}\right]=\left[\begin{array}{l}
I_{1} \\
\vdots \\
I_{n}
\end{array}\right]^{T} \bullet R_{n} \bullet\left[\begin{array}{l}
I_{1} \\
\vdots \\
I_{n}
\end{array}\right]
$$

4) Maximum and minimum power losses analysis

Head end current $I_{0}$ changes from zero to the maximum $I_{\Sigma m}$ with a set step-size $\Delta I_{k}$. When changes to the L step, $I_{0}=\sum_{k=1}^{L} \Delta I_{k}$, and substitute it into formula of $P$.

$$
P=\left[\begin{array}{c}
I_{1} \\
\vdots \\
I_{n}
\end{array}\right]^{T} \bullet R_{n} \bullet\left[\begin{array}{c}
I_{1} \\
\vdots \\
I_{n}
\end{array}\right]
$$

By using flexible tolerance polyhedron method, the maximum and minimum power losses of head end current are $P_{\max }\left(\sum_{k=1}^{L} \Delta I_{k}\right)$ and $P_{\min }\left(\sum_{k=1}^{L} \Delta I_{k}\right)$.

5) Maximum and minimum equivalent resistances analysis

According to the maximum and minimum power losses and the corresponding head end currents, the maximum and minimum equivalent resistances can be calculated, as in (4) and (5).

$$
\begin{gathered}
R_{\max }\left(\sum_{k=1}^{L} \Delta I_{k}\right)=\frac{P_{\max }\left(\sum_{k=1}^{L} \Delta I_{k}\right)}{\left(\sum_{k=1}^{L} \Delta I_{k}\right)^{2}} \\
R_{\min }\left(\sum_{k=1}^{L} \Delta I_{k}\right)=\frac{P_{\min }\left(\sum_{k=1}^{L} \Delta I_{k}\right)}{\left(\sum_{k=1}^{L} \Delta I_{k}\right)^{2}}
\end{gathered}
$$

6) Linear interpolation functions of the maximum and minimum equivalent resistances analysis

When head end current $I_{0}$ changes to the L step, this L step size's left end point head end current is $I_{0}=\sum_{k=1}^{L-1} \Delta I_{k}$, corresponding to this, the maximum and minimum equivalent resistances are $R_{\max }\left(\sum_{k=1}^{L-1} \Delta I_{k}\right)$ and $R_{\min }\left(\sum_{k=1}^{L-1} \Delta I_{k}\right)$. This L step size's right end point head end current is $I_{0}=\sum_{k=1}^{L} \Delta I_{k}$, and corresponding to this, the maximum and minimum equivalent resistances are $R_{\max }\left(\sum_{k=1}^{L} \Delta I_{k}\right)$ and $R_{\min }\left(\sum_{k=1}^{L} \Delta I_{k}\right)$. The linear interpolation functions of the maximum and minimum equivalent resistances can be expressed, as in (6) and (7).

$$
\begin{array}{ll}
R_{\max }\left(I_{0}\right)=a_{\max L}+b_{\max L} \cdot I_{0} & \sum_{k=1}^{L-1} \Delta I_{k} \leq I_{0} \leq \sum_{k=1}^{L} \Delta I_{k} \\
R_{\min }\left(I_{0}\right)=a_{\min L}+b_{\min L} \cdot I_{0} & \sum_{k=1}^{L-1} \Delta I_{k} \leq I_{0} \leq \sum_{k=1}^{L} \Delta I_{k}
\end{array}
$$


Where $a_{\max L}, b_{\max L}, a_{\min L}$ and $b_{\min L}$ can be calculated, as in (8), (9), (10) and (11).

$$
\begin{aligned}
& a_{\max L}=\frac{R_{\max }\left(\sum_{k=1}^{L-1} \Delta I_{k}\right) \cdot \sum_{k=1}^{L} \Delta I_{k}-R_{\max }\left(\sum_{k=1}^{L} \Delta I_{k}\right) \cdot \sum_{k=1}^{L-1} \Delta I_{k}}{\Delta I_{L}} \\
& b_{\max L}=\frac{R_{\max }\left(\sum_{k=1}^{L} \Delta I_{k}\right)-R_{\max }\left(\sum_{k=1}^{L-1} \Delta I_{k}\right)}{\Delta I_{L}} \\
& a_{\min L}=\frac{R_{\min }\left(\sum_{k=1}^{L-1} \Delta I_{k}\right) \cdot \sum_{k=1}^{L} \Delta I_{k}-R_{\max }\left(\sum_{k=1}^{L} \Delta I_{k}\right) \cdot \sum_{k=1}^{L-1} \Delta I_{k}}{\Delta I_{L}} \\
& b_{\min L}=\frac{R_{\min }\left(\sum_{k=1}^{L} \Delta I_{k}\right)-R_{\min }\left(\sum_{k=1}^{L-1} \Delta I_{k}\right)}{\Delta I_{L}}
\end{aligned}
$$

7) Maximum and minimum energy consumption within a given period of time analysis

According to the linear interpolation functions of the maximum and minimum equivalent resistances, actual measurement of head end three-phase currents, the maximum and minimum equivalent resistances can be calculated.

Assume that total dead loss is $P_{\Sigma 0}(\mathrm{~kW})$, head end maximum current is $I_{\sum m}(\mathrm{~A})$. There are M segments linear interpolation functions of the maximum and minimum equivalent resistances from 0 to $I_{\sum m}$, and single phase linear interpolation function coefficient of maximum equivalent resistance is $a_{\max L}(\Omega, \mathrm{L}=1 \ldots \mathrm{M})$.

Calculation start from 0 o'clock, 0 minute and 0 second of someday. In any time segment $\mathrm{i}$, measure three-phase current $I_{a i}, I_{b i}$ and $I_{c i}$ at the same time with sample interval $\Delta T(\mathrm{~s})$.

Then the maximum energy consumption $\Delta A_{N \text { max }}(\mathrm{kWh})$ from the beginning to the current segment $\mathrm{N}$ cam be calculated, as in (12).

$$
\Delta A_{N \max }=\frac{\Delta T}{36 \cdot 10^{5}} \sum_{i=1}^{N}\left(I_{a i}^{2} \cdot R_{\max }\left(I_{a i}\right)+I_{b i}^{2} \cdot R_{\max }\left(I_{b i}\right)+I_{c i}^{2} \cdot R_{\max }\left(I_{c i}\right)\right)+\frac{N \cdot \Delta T}{3600} \cdot P_{\Sigma 0}
$$

Then the minimum energy consumption $\Delta A_{N \text { min }}(\mathrm{kWh})$ from the beginning to the current segment $\mathrm{N}$ cam be calculated, as in (13).

$$
\Delta A_{N \text { min }}=\frac{\Delta T}{36 \cdot 10^{5}} \sum_{i=1}^{N}\left(I_{a i}^{2} \cdot R_{\min }\left(I_{a i}\right)+I_{b i}^{2} \cdot R_{\min }\left(I_{b i}\right)+I_{c i}^{2} \cdot R_{\min }\left(I_{c i}\right)\right)+\frac{N \cdot \Delta T}{3600} \cdot P_{\Sigma 0}
$$

\section{Weak Line Identification Method}

\subsection{Energy Consumption Interval Partition}

According to the six indexes of the weak line identification, energy consumption in $10 \mathrm{kV}$ distribution network can be divided into 4 intervals: unreasonable loss interval, high loss interval, reactive power management interval, low loss interval.

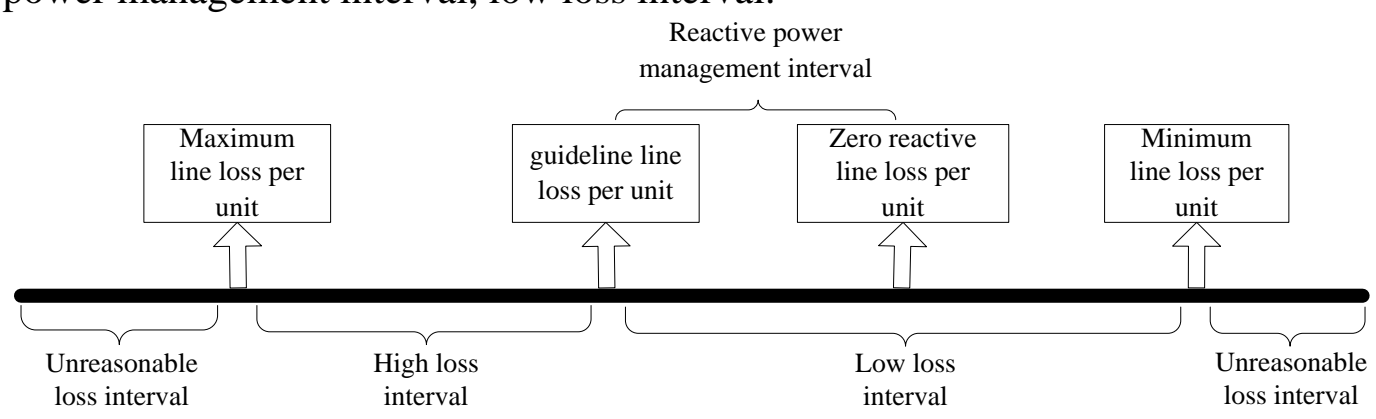

Fig. 1 Energy consumption interval partition 
Maximum power loss electric quantity and maximum line loss per unit can be the upper limit value of energy consumption. When measurement maximum value is very big, consider that whether or not the line load of transformer or line is heavier, power supply radius is too big, the energy-intensive transformer is running. Transform in time according to specific circumstances to reduce the line loss.

When guideline loss electric quantity and guideline line loss per unit are very big, consider that whether or not the line load of transformer or line is heavier, power supply radius is too big, the energy-intensive transformer is running.

Minimum power loss electric quantity and minimum line loss per unit can be the lower limit value of energy consumption.

When guideline loss electric quantity and guideline line loss per unit is much larger than zero reactive loss electric quantity and zero reactive line loss per unit, consider that reactive power compensation should be reasonable, and the management of putting into or back operation of reactive power compensation equipment should be strengthened, in order to reduce line loss.

When three-phase current harmonic total cumulative rate is very large, harmonic suppression should be strengthened, in order to reduce the line loss.

When un-balanced load cumulative rate is very large, the reason of un-balanced load should be found, and adjust the three-phase load to balance, in order to reduce line loss.

\subsection{Weak Line Screening}

In unreasonable loss interval: weak line screening when statistics value is larger than the maximum value.

Step 1: Check whether lines, transformers and section switches were changed. If changed, reset the setting parameters. If not, then go to step 2.

Step 2: Check whether the line loss statistics are correct. If not, should be correct in time, in order to reduce line loss. If it's right, then go to step 3.

Step 3: Check whether the lines or transformers are overload, if it is, should be eliminated in time, in order to reduce line loss. If not, then go to step 4.

Step 4: Check whether the harmonic content is too large by total harmonic contain accumulation rate, if harmonic content is big, it should be timely governance in order to reduce line loss, if harmonic content is small, then go to step 5 .

Step 5: Check whether there is stolen, the leakage of electric situation. If exist, should be correct in time, in order to reduce line loss. If not, then go to step 6.

Step 6: Check whether there is a measurement error. If exist, should be correct in time, in order to reduce line loss.

In unreasonable loss interval: weak line screening when statistics value is less than the minimum value.

Step 1: Check whether lines, transformers and section switches were changed. If changed, reset the setting parameters. If not, then go to step 2.

Step 2: Check whether the line loss statistics are correct. If not, should be correct in time, in order to reduce line loss. If it's right, then go to step 3.

Step 3: Check whether there is a measurement error. If exist, should be correct in time, in order to reduce line loss.

In reasonable loss interval: weak line screening when statistics value is in the high loss interval.

Find the reason from operation mode (including load distribution), the types of transformer (whether or not energy-saving transformer) line route and other respects to do the lean management.

In reasonable loss interval: weak line screening when statistics value is in the reactive power management interval.

The ratio of zero reactive line loss per unit and guideline line loss per unit, especially the ratio of zero reactive line loss per unit per hour and guideline line loss per unit per hour, can indirectly reflect the reactive power operation and management level, and adopt the loss reduction measures according to the circumstance. The closer the ratio of each hour is to 1 , the better the situation of the reactive power operation and management, And vice versa.

In reasonable loss interval: weak line screening when statistics value is in the low loss interval. 
Line loss management is good, generally further loss reduction management does not need.

\section{Example Analysis}

\subsection{Compared with the Results of Meter Reading}

Choose one $10 \mathrm{kV}$ distribution network line of Hubei province as the example to compare with the results of meter reading.

Table 1 Line loss per unit

\begin{tabular}{ccccc}
\hline Meter reading & Maximum & Minimum & Guideline & Zero- reactive \\
\hline $9.49 \%$ & $9.14 \%$ & $6.72 \%$ & $8.31 \%$ & $8.05 \%$ \\
\hline
\end{tabular}

Table 1 shows that meter reading line loss per unit is larger than the maximum line loss per unit. According to the weak line screening steps, find that, energy meter input the wrong number of transformation ratio of current transformer in meter reading system. After modification, the meter reading line loss per unit is $8.72 \%$. Compared in 4 days, results show the veracity.

\subsection{Loss Reduction Project Example}

Choose 5 typical lines in Hubei province as the example to compare the application results with the results of meter reading. The total data of 5 lines are showed in table 2.

Table 2 Total data of 5 lines

\begin{tabular}{ccccccc}
\hline Line & $\begin{array}{c}\text { Total power } \\
\text { supply } \\
(\mathrm{kWh})\end{array}$ & $\begin{array}{c}\text { Total meter } \\
\text { reading line } \\
\text { loss per unit } \\
(\%)\end{array}$ & $\begin{array}{c}\text { Total meter } \\
\text { reading loss } \\
\text { electric } \\
\text { quantity } \\
(\mathrm{kWh})\end{array}$ & $\begin{array}{c}\text { Total } \\
\text { maximum } \\
\text { line loss per } \\
\text { unit }(\%)\end{array}$ & $\begin{array}{c}\text { Total } \\
\text { maximum } \\
\text { loss electric } \\
\text { quantity } \\
(\mathrm{kWh})\end{array}$ & $\begin{array}{c}\text { Total loss } \\
\text { reduction } \\
\text { electric } \\
\text { quantity } \\
(\mathrm{kWh})\end{array}$ \\
\hline L1 & 12724654 & 8.55 & 1087958 & 4.85 & 617146 & 470812 \\
L2 & 7166350 & 15.89 & 1139059 & 13.39 & 959574 & 179485 \\
L3 & 9027588 & 7.88 & 711373 & 6.45 & 582279 & 129094 \\
L4 & 12203617 & 8.03 & 979950 & 5.98 & 729776 & 250174 \\
L5 & 6662556 & 7.80 & 519679 & 6.00 & 399753 & 119926 \\
\hline
\end{tabular}

Table 2 shows that the meter reading line loss per units of 5 lines are all larger than their maximum line loss per units, which declare the maximum line loss per unit is the assessment line loss per unit. And from the data of total loss reduction electric quantity, the total electric quantities of 5 lines can be $114949 \mathrm{kWh}$ each year. After contrastive analysis, the reasons of the high loss of 5 lines were found, and the following technical renovation measures can be taken: about L1 and L2, load supply and meter reading should be in the same time; about L3, wires between two transformers should change to the aluminum wires with sectional area of $70\left(\mathrm{~mm}^{2}\right)$; about L4 and L5, S11 energy-saving transformers should be added in the distribution network.

\section{Summary}

The weak line identification method of energy consumption in $10 \mathrm{kV}$ distribution network is studied to change the uncertain line loss meter reading value to a certain scope, which can help overcome the topological structure of complex and the shortage of that measuring equipment are not complete in $10 \mathrm{kV}$ distribution network. It builds a new weak line identification indexes system of 6 indexes to divide the line losses into 4 intervals. According to the intervals, the weak line of energy consumptions can be found, which helps find the reason of power losses so as to guild the management and technical methods of loss reductions. 


\section{References}

[1]. DL/T686-1999,Electric energy loss calculation guide rule in power grid [S].

[2]. Lu Guangxiang,Shan Yuanda,Gong Lenian,et al.The method about the determination of maximum load's loss hours is doubtful [J].Transactions of China Electrotechnical Society,1996, 2(11):54-59(in Chinese).

[3]. Yuan Huimei,Guo Xiqing, Yu Haibo.New method for calculating energy losses in medium-voltage distribution systems [J].Automation of Electric Power Systems , 2002, 26(11): 50-53.

[4]. Ding Xinhai,Luo Yifang,Liu Wei et al.A new practical method for calculating line loss of distribution network-improved iteration method[J].Power System Technology, 2000, 24(1): 39-42.

[5]. Liu Jun,Qian Qi,Liu Haitao,et al.Design and research on $10 \mathrm{kV}$ distribution network data acquisition and power loss automatic system [J].Power System Technology, 2006, 30(10): 571-574(in Chinese).

[6]. Yu Weiguo,Xiong Youjing,Zhou Xinfeng,et al.Analysis on technical line losses of power grids and countermeasures to reduce line losses [J].Power System Technology,2006,30(18):54-63(in Chinese). 